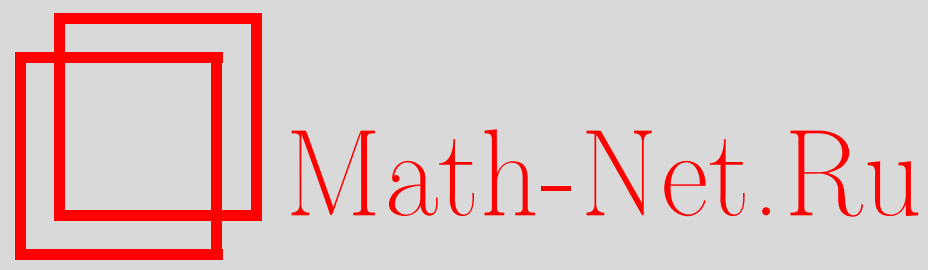

В. Г. Курбатов, Замечание о предельных операторах, Функи. анализ и его прил., 1996, том 30, выпуск 1, 73-75

DOI: https://doi.org/10.4213/faa510

Использование Общероссийского математического портала MathNet.Ru подразумевает, что вы прочитали и согласны с пользовательским соглашением

http://www . mathnet.ru/rus/agreement

Параметры загрузки:

IP : 54.205 .225 .156

26 апреля 2023 г., 16:28:01

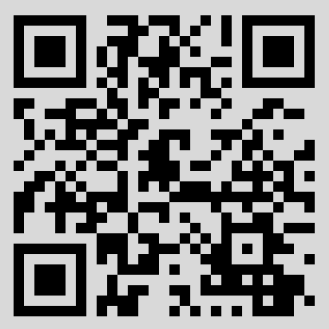


занные с ней вопросы теории представлений. Физматгиз, М. (1962). 5. Стейн И., Вейс $Г$. Введение в гармонический анализ на евклидовых пространствах. Мир, M. (1974).

Институт математики

Поступило в редакцию НАН Украины

8 апреля 1994 г. В переработанном виде 2 октября 1995 г.

УдК 517.9

\title{
Замечание о предельных операторах
}

\author{
(C) $1996 . \quad$ В. Г. КурьАтов
}

Настоящая заметка тесно связана с работами [1-3]. В ней результат статьи [1] частично переносится на случай пространств $L_{p}(p \neq 2)$ и операторов общего вида (подробнее см. ниже).

Пусть $E$ - конечномерное комплексное банахово пространство и $\Omega \subset \mathbb{R}^{n}$ измеримое подмножество. Обозначим через $L_{p}(\Omega)$ пространство классов совпадающих почти всюду измеримых функций $u: \Omega \rightarrow E$ с соответствующей нормой; положим $L_{p}=L_{p}\left(\mathbb{R}^{n}\right)$.

Для $x, \xi \in \mathbb{R}^{n}$ символом $\langle\xi, x\rangle$ будем обозначать скалярное произведение. Для $\xi \in \mathbb{R}^{n}$ положим $\left(\Psi_{\xi} u\right)(x)=e^{i\langle\xi, x\rangle} u(x)$. Пусть $T: L_{p}(\Omega) \rightarrow L_{p}$ - линейный ограниченный оператор. Оператор $\widetilde{T}$ назовем правылм предельнылм для $T$, если существует такая последовательность $\xi_{k} \in \mathbb{R}^{n}$, что $\left|\xi_{k}\right| \rightarrow+\infty$ и последовательность $T\left\{\xi_{k}\right\}=\Psi_{\xi_{k}}^{-1} T \Psi_{\xi_{k}}$ сильно (т. е. на любом $\left.u \in L_{p}(\Omega)\right)$ сходится к $\widetilde{T}$.

Положим

$$
|T|_{+}=\inf \{\|T u\|:\|u\|=1\}, \quad|T|_{\lambda+}=\sup \left\{\left|T: M \rightarrow L_{p}\right|_{+}: \operatorname{codim} M<\infty\right\}
$$

(имеется в виду, что $M$ пробегает всевозможные замкнутые подпространства $L_{p}(\Omega)$ конечной коразмерности). Очевидно, что $|T|_{+}>0$ тогда и только тогда, когда ядро оператора $T$ состоит из нуля, а его образ замкнут, и $|T|_{\lambda+}>0$ тогда и только тогда, когда ядро оператора $T$ конечномерно, а его образ замкнут. Величина $|T|_{+}$введена в [4], по поводу величины $|T|_{\lambda+}$ см. [5].

TеОрема 1. Пусть $p<\infty, T: L_{p}(\Omega) \rightarrow L_{p}$ - линейньий ограниченный оператор и $\widetilde{T}-$ правый предельный оператор для $T$. Тогда $|T|_{\lambda+} \leqslant|\widetilde{T}|_{+}$.

В статьях [1-3] наряду с описанными выше «частотными» предельными операторами рассматриваются также «координатные» предельные операторы, определяемые как пределы последовательностей $S_{h_{k}}^{-1} T S_{h_{k}}$, где $\left(S_{h} u\right)(x)=u(x-h)$. В $[1,2]$ установлено, что для псевдодифференциальных операторов в пространстве $L_{2}[1]$ и в некоторых других специальных пространствах [2] фредгольмовость эквивалентна обратимости всех "частотно-координатных" предельных операторов. В приводимом ниже следствии формулируется лишь половина «частотной» части этого утверждения, но для более широкого класса операторов 
и чуть более общего понятия предельного оператора. Перенесению «координатной» части результатов работ $[1,2]$ на случай операторов общего вида посвящена статья [3].

Ниже будем предполагать, что $T$ - линейный ограниченный оператор, действуюший из $L_{p}$ в $L_{p}$.

Оператор $\widetilde{T}$ назовем двусторонним предельным для $T$, если существует такая последовательность $\xi_{k} \in \mathbb{R}^{n}$, что $\left|\xi_{k}\right| \rightarrow+\infty, T\left\{\xi_{k}\right\}$ сильно сходится к $\widetilde{T}$, а сопряженная последовательность $T\left\{\xi_{k}\right\}^{*}$ сильно сходится к $\widetilde{T}^{*}$.

СледСтвИЕ. Пусть $1<p<\infty$ и оператор $T$ фредгольмов. Тогда все двусторонние предельные операторы $\widetilde{T}$ обратимы, причем нормы обратных равномерно ограничены:

$$
\left\|\widetilde{T}^{-1}\right\| \leqslant 1 /|T|_{\lambda+} .
$$

Обозначим через $L_{p_{\Omega}}$ подпространство пространства $L_{p}$, состоящее из функций, носители которых лежат в $\Omega \subset \mathbb{R}^{n}$. Очевидно, что $L_{p_{\Omega}}$ канонически изоморфно $L_{p}(\Omega)$. Положим $|T|_{\Lambda+}=\inf \left|T: L_{p_{\Omega}} \rightarrow L_{p}\right|_{\lambda+}$, где инфимум берется по всем ограниченным измеримым подмножествам $\Omega \subset \mathbb{R}^{n}$.

Tеорема 2. Пусть $p<\infty, T: L_{p} \rightarrow L_{p}$ и $\widetilde{T}$ - правый предельный опеpamop. Тогда $|T|_{\Lambda+} \leqslant|\widetilde{T}|_{+}$.

Оператор $T$ назовем локально фредгольмовым (подробнее см. [6]), если $|T|_{\Lambda+}>0$ и $\left|T^{*}\right|_{\Lambda+}>0$. Будем говорить, что линейный ограниченный оператор $K: L_{p} \rightarrow L_{p}$ является локально компактным, если для любого ограниченного измеримого подмножества $\Omega \subset \mathbb{R}^{n}$ сужения $K: L_{p_{\Omega}} \rightarrow L_{p}$ и $K^{*}: L_{p_{\Omega}}^{*} \rightarrow L_{p}^{*}$ являются компактными операторами. Очевидно, если $K$ локально компактен, то $1+K$ локально фредгольмов. Простейший пример локально компактного оператора в $L_{p}(1<p<\infty)$ - оператор свертки с функцией класса $L_{1} \cap L_{\infty}$.

СледСтвИЕ. Пусть $1<p<\infty$ и оператор $T: L_{p} \rightarrow L_{p}$ является локально фредгольмовым. Тогда все двусторонние предельные операторы $\widetilde{T}$ для Т обратимы, причем нормы обратных равномерно ограничены:

$$
\left\|\widetilde{T}^{-1}\right\| \leqslant 1 /|T|_{\Lambda+} .
$$

ПримеР. Пусть $n=1, E=\mathbb{C}, 1<p<\infty$ и

$$
(T u)(x)=\sum_{m=1}^{\infty} a_{m}(x) u\left(x-h_{m}\right)+\sum_{m=1}^{\infty} b_{m}(x)(H u)\left(x-h_{m}\right)+(K u)(x),
$$

где

$$
(H u)(x)=\frac{1}{i \pi} \int_{-\infty}^{+\infty} \frac{u(s)}{s-x} d s
$$

- оператор Гильберта, $K$ - локально компактный оператор, $h_{m} \in \mathbb{R}, a_{m}$ и $b_{m}$ - функции класса $L_{\infty}$, причем $b_{m}$ непрерывно дифференцируемы, а

$$
\sum_{m=1}^{\infty}\left\|a_{m}\right\|_{L_{\infty}}+\sum_{m=1}^{\infty}\left\|b_{m}\right\|_{L_{\infty}}<\infty
$$


Из непрерывной дифференцируемости коэффициентов $b_{m}$ следует, что сопряженный к $T$ имеет такой же вид, что и $T$. Нетрудно видеть, что операторы

$$
\left(D_{ \pm} u\right)(x)=\sum_{m=1}^{\infty}\left[a_{m}(x) \pm b_{m}(x)\right] u\left(x-h_{m}\right)
$$

являются двусторонними предельными для $T$. Поэтому из следствия теоремы 2 вытекает, что необходимым условием локальной фредгольмовости оператора $T$ является обратимость операторов $D_{+} u D_{-}$. Аналогичное явление имеет место и при замене $H$ некоторыми сингулярными интегралами [7] в $\mathbb{R}^{n}$.

\title{
ЛиТеРАтУРА
}

1. Ланге Б. В., Рабинович В. С. Матем. сб., 129, 175-185 (1986). 2. Рабинович В. С. Сиб. матем. ж., 19, 149-161 (1988). 3. Рабинович В. С. Матем. заметки, 51, 90-101 (1992). 4. Gindler H. A., Taylor A. E. Studia Math., 22, 15-41 (1962/63). 5. Ахмеров P. P. и др. Меры некомпактности и уплотняющие операторы. Наука, Новосибирск (1986). 6. Курбатов В. Г. Линейные дифференциально-разностные уравнения. Изд-во Воронежского гос. ун-та (1990). 7. Стейн И. Сингулярные интегралы и дифференциальные свойства функций. Мир, М. (1973).

Воронежский государственный

Поступило в редакцию университет

12 мая 1994 г.

УдК 517.986

\section{Эпиморфизмы и разреженные подмножества банаховой алгебры}

\author{
(c) 1996. Ф. Г. МАКСудов, Н. М. САдиков, Ю. В. Туровский ${ }^{1}$
}

В работе рассматриваются условия разреженности образа операторов, ассоциированных с эпиморфизмом банаховой алгебры. Ранее разреженность в смысле принадлежности множеству квазинильпотентных элементов рассмотрена для дифференцирований в [1-4] и др. В данной работе в качестве эталона для разреженности образа берутся подмножества элементов банаховой алгебры с конечным спектром.

Полученные результаты можно охарактеризовать следующим образом. Эпиморфизмы, удовлетворяющие условию разреженности образа ассоциированных с ними операторов, или некоторые их степени должны индуцироваться алгебраическими операторами во всех неприводимых представлениях, кроме, возможно, конечного числа конечномерных.

1. Пусть $A-$ унитальная комплексная банахова алгебра, $\operatorname{Rep} A-$ множество всех ее представлений линейными операторами, $\operatorname{Irr} A-$ множество всех неприводимых представлений и Fin $A$ - множество всех конечных прямых сумм

${ }^{1}$ Третий из авторов благодарит Международный фонд «Культурная инициатива»и Академию естественных наук России за поддержку. 